\title{
EFECTO DE UNA INTERVENCIÓN EDUCATIVA SOBRE HIGIENE ALIMENTARIA A TRAVÉS DEL CONOCIMIENTO Y PRÁCTICA DE MADRES DEL CENTRO POBLADO CHACARITA - SUNAMPE CHINCHA PRIMER TRIMESTRE 2014.
}

Effect of an educational intervention on food hygiene through knowledge and practice of mothers Chacarita town center - first quarter 2014 Sunampe Chincha.

Viviana Loza Félix ${ }^{1, a}$, Marianela Clarisa Pecho Tataje ${ }^{2, a, b}$, Cecilia Paquita Uribe Quiroz ${ }^{2, a, b}$, Dennis Lévano García ${ }^{c}$

${ }^{1}$ Universidad Nacional San Luis Gonzaga de Ica, Facultad de enfermería - Perú.

${ }^{2}$ Hospital Augusto Hernández Mendoza.

${ }^{\mathrm{a}}$ Lic. en Enfermería, ${ }^{\mathrm{b}} \mathrm{Mg}$. en Educación Superior, ${ }^{\mathrm{c}}$ Bach. en Enfermería

\section{RESUMEN}

La higiene alimentaria comprende todas las medidas necesarias para garantizar la inocuidad sanitaria de los alimentos, manteniendo a la vez el resto de cualidades que les son propias, con especial atención al contenido nutricional, constituyendo una de las principales causas de enfermedades prevalentes en la población infantil. Objetivo: Determinar el efecto de una intervención educativa sobre higiene alimentaria a través del conocimiento y práctica de madres del centro poblado Chacarita - Sunampe Chincha primer trimestre 2014. Material y métodos: El estudio fue cuantitativo, descriptivo y de corte transversal, la técnica utilizada fue la encuesta y el instrumento un cuestionario de opción múltiple para la primera variable y observación para la segunda variable cuyo instrumento fue una guía de observación, previamente validado. Resultados: El conocimiento sobre higiene alimentaria antes de la intervención educativa fue deficiente en un $93 \%$ y posterior a la intervención fue óptimo en el $100 \%$ de las madres. La práctica de higiene alimentaria antes de la intervención educativa fue inadecuada en un $100 \%$, sin embargo después de la intervención alcanzó 53\% adecuada. Conclusiones: El efecto de la intervención educativa fue positivo, demostrado a través del valor de T Student $(4,18)$

Palabras Clave: Intervención educativa, higiene alimentaria, conocimiento, práctica.

\section{SUMMARY}

Food hygiene comprises all necessary measures to ensure the health safety of foods, while maintaining the other qualities that are proper, with particular attention to nutritional content, constituting one of the main causes of diseases prevalent in children. Objective: To determine the effect of an educational intervention on food hygiene through knowledge and practice of mothers Chacarita population center - Chincha Sunampe first quarter 2014. Methods: The study was quantitative, descriptive, cross-sectional, the technique used was the survey instrument and a multiple-choice for the first variable and the second variable observation whose instrument was an observation guide, previously validated. Results: Knowledge of food hygiene before the educational intervention was poor in $93 \%$ and after the intervention was excellent in $100 \%$ of mothers. The practice of food hygiene before the educational intervention was inappropriate in $100 \%$, but after the intervention was $53 \%$ right. Conclusions: The effect of the educational intervention was positive, demonstrated through the Student $T$ value $(4,18)$

Keywords: Educational intervention, food hygiene, knowledge, practice. 


\section{INTRODUCCIÓN}

La insalubridad de los alimentos ha representado un problema de salud para el ser humano desde los albores de la historia, y muchos de los problemas actuales en esta materia no son nuevos. Aunque los gobiernos de todo el mundo se están esforzando al máximo por aumentar la salubridad del suministro de alimentos, la existencia de enfermedades de transmisión alimentaria sigue siendo un problema de salud significativo tanto en los países desarrollados como en los países en desarrollo. La organización mundial de la salud (OMS) es consciente desde hace tiempo de la necesidad de concienciar a los manipuladores de alimentos sobre sus responsabilidades respecto de la inocuidad de éstos; se ha calculado que cada año mueren 1,8 millones de personas como consecuencia de enfermedades diarreicas, cuya causa puede atribuirse en la mayoría de los casos a la ingesta de agua o alimentos contaminados. Una preparación adecuada de los alimentos puede prevenir la mayoría de las enfermedades de transmisión alimentaria (1). En los países de la Unión Europea y de Occidente en general las tres primeras premisas se alcanzan de forma generalizada, salvo excepciones ocasionales, por lo que es el último punto, el que se refiere a la calidad y la inocuidad de los alimentos, el que cobra relevancia y protagonismo actual y al que van dirigidas la mayoría de las políticas vinculadas a la seguridad alimentaria. Adicionalmente en estos países desarrollados se está poniendo de manifiesto la existencia de un nuevo problema nutricional de primer orden, representado por el excesivo consumo de alimentos que resulta en el sobrepeso y la obesidad. Por el contrario, en los países en desarrollo y en los organismos multilaterales, el concepto de seguridad alimentaria se interpreta en su definición más amplia y con un mayor énfasis en su relación con la pobreza y la falta de un acceso adecuado a una alimentación suficiente por parte de los sectores más pobres de la población (2).
Según el Ministerio de Salud (MINSA) en el año 2012, la incidencia de enfermedades infecciosas intestinales en la población infantil alcanzo $7,9 \%$, caracterizado por diarreas, siendo la segunda causa de morbilidad; continuando dicha tendencia en el primer trimestre 2013 (3). De acuerdo al informe electrónico del programa nacional Wawa Wasi donde su implementación tiene como prioridad la alimentación, cuyas madres cuidadoras tiene por objetivo el aprendizaje de conductas saludables monitorizadas revelan que el $92,37 \%$ de los niños y las niñas que asisten al wawa wasi han adquirido los hábitos básicos de salud esperados para su edad: lavado de cara y manos, cepillado de dientes y el $93,2 \%$ de Madres Cuidadoras realiza adecuadamente las prácticas saludables en el wawa wasi donde se incluye la higiene alimentaria (4).

Según Puentes (2010) en una investigación hecha en Cuba, en un grupo de madres, se obtuvo que el nivel de conocimiento de las madres, al inicio del estudio, sobre nutrición en preescolares con diarreas fue malo $(55,3 \%)$. Después de aplicado el programa educativo el nivel de conocimiento fue evaluado de bueno en el $87,0 \%$. (5). Asimismo, en un estudio realizado por Chaves (2011) en Ecuador, se obtuvo como resultado que el conocimiento respecto a la higiene la preparación de alimentos fue deficiente en un $69,3 \%$, otras conductas relacionadas a la higiene alimentaria como el lavado de manos era realizado solo tres veces en el día en las personas encargadas de cocinar los alimentos. (6).

La investigación realizada es importante porque abarca dos aspectos básicos en el cuidado de la salud como son la higiene y la alimentación, el conocimiento y práctica de ambos aspectos se verán reflejadas a través de la higiene alimentaria, conducta adquirida en las madres que al ser saludables aseguren la salud de los niños y comunidad en general. El profesional de enfermería tiene la labor de prevenir y promocionar utilizando diversas 
estrategias como intervenciones educativas basadas en la información, dando modelos y experiencias sobre la alimentación e higiene de los alimentos como parte de sus roles en la educación sanitaria en el primer nivel de atención de salud. Las intervenciones educativas constituyen estrategias eficaces en el aprendizaje de temas de interés en la salud dirigida a motivar estilos de vida saludables siendo las madres el ente multiplicador en la salud de la familia. En el estudio se planteó como objetivo determinar el efecto de una intervención educativa sobre higiene alimentaria a través del conocimiento y práctica de madres del centro poblado Chacarita - Sunampe Chincha primer trimestre 2014.

\section{MATERIAL Y METODOS:}

La investigación es de tipo cuantitativo, diseño cuasi experimental, longitudinal La población está constituida por la totalidad de madres del centro poblado Chacarita Sunampe las cuales suman 220 madres de niños menores de 5 años según información del agente comunitario y gobernador del sector. La Muestra fue intencional a criterio del investigador la cual fue de 15 madres de acuerdo a criterios de inclusión y exclusión. La técnica utilizada fue la encuesta para la medición del conocimiento y como instrumento un cuestionario y para la práctica se utilizó una guía de observación, ambos instrumentos validados a través de juicio de expertos. Los datos se procesaron mediante el programa de Microsoft Excel y Word versión 2010. Para la presentación de datos se emplearon tablas estadísticas con valores porcentuales y los gráficos de barras, lo cual permitió el respectivo análisis. Para demostrar el efecto de la intervención se tomó en cuenta el estadístico t de Student.

\section{RESULTADOS}

La edad de las madres adolescentes en su mayoría fue de 16 años alcanzando 33,3\% la ocupación que tenían fue ama de casa al $100 \%$, el estado civil fue conviviente en el $60 \%$, su grado de instrucción fue secundaria completa en el $66,7 \%$ y un $73,3 \%$ refiere que no recibió información sobre higiene alimentaria. (Tabla 1). Se puede observar que de las madres encuestadas con respecto al conocimiento global de higiene alimentaria antes de las intervenciones educativas el 93\% fueron deficientes y $7 \%$ fueron optimas, después de las intervenciones dio como resultado que el $0 \%$ fueron deficientes y el $100 \%$ fueron óptimas. (Tabla 2) La práctica de higiene alimentaria antes de la intervención educativa fue inadecuada en el $100 \%$ de madres, sin embargo después de la intervención educativa fue adecuado en el $53 \%$ de las madres. (Tabla 3 )

TABLA 1. DATOS GENERALES DE LAS MADRES DEL CENTRO POBLADO CHACARITA - SUNAMPE, CHINCHA PRIMER TRIMESTRE 2014.

\begin{tabular}{ccrr}
\hline $\begin{array}{c}\text { DATOS } \\
\text { GENERALES }\end{array}$ & CATEGORIAS & $\mathbf{f}$ & $\%$ \\
\hline EDAD & 15 años & 1 & 6,7 \\
& 16 años & 5 & 33,3 \\
& 17 años & 3 & 13,3 \\
& 18 años & 3 & 13,3 \\
& 19 años & 3 & 13,3 \\
Ocupación & Ama de casa & 15 & 100,0 \\
Estado civil & Conviviente & 9 & 60,0 \\
& Casada & 5 & 33,3 \\
Grado de & Separada & 1 & 6,7 \\
instrucción & Secundaria & 10 & 66,7 \\
& completa & & \\
Recibió & Secundaria & 5 & 33,3 \\
información & incompleta & 11 & 73,3 \\
& No & 4 & 26,7 \\
\hline & Total & 15 & 100,0 \\
\hline
\end{tabular}


TABLA 2. CONOCIMIENTO SOBRE HIGIENE ALIMENTARIA EN MADRES DEL CENTRO POBLADO CHACARITA SUNAMPE, CHINCHA.

\begin{tabular}{clrr}
\hline CONOCIMIENTO SOBRE HIGIENE ALIMENTARIA & $\mathbf{f}$ & $\%$ \\
\hline \multirow{2}{*}{ ANTES } & DEFICIENTE & 14 & 93 \\
& ÓPTIMO & 1 & 7 \\
DESPUES & DEFICIENTE & 0 & 0 \\
TOTAL & ÓPTIMO & 15 & 100 \\
\hline
\end{tabular}

TABLA 3. PRÁCTICA SOBRE HIGIENE ALIMENTARIA EN MADRES DEL CENTRO POBLADO CHACARITA - SUNAMPE, CHINCHA.

\begin{tabular}{clcc}
\hline PRÁCTICA SOBRE HIGIENE ALIMENTARIA & $\mathbf{f}$ & $\%$ \\
\hline \multirow{2}{*}{ ANTES } & INADECUADO & 15 & 100 \\
& ADECUADO & 0 & 0 \\
DESPUES & INADECUADO & 7 & 47 \\
TOTAL & ADECUADO & 8 & 53 \\
\hline
\end{tabular}

\section{DISCUSIÓN:}

Una de las estrategias que utiliza el profesional de enfermería para fortalecer la educación de la población es la intervención educativa, el estudio realizado refleja una experiencia relacionada a la higiene alimentaria en madres adolescentes.

La edad de las madres adolescentes en su mayoría fue de $33,3 \%$ la ocupación que tenían fue ama de casa al $100 \%$, el estado civil fue conviviente en el $60 \%$, su grado de instrucción fue secundaria completa en el $66,7 \%$ y un $73,3 \%$ refiere que no recibió información sobre higiene alimentaria. Los resultados difieren con los de García $R$, Casado C, Pérez A, Sosa I. quienes en su estudio encontraron dentro de su grupo de estudio predominante las edades entre $17 \mathrm{y}$ 21 años en un 53,5\% (7). Matta W, Ortega D, en su estudio encontraron que el grado de instrucción de la madre fue secundaria y la ocupación era ama de casa (8). Como se puede observar la similitud con el estudio citado destacándose que las madres en estudio cuentan con una fortaleza respecto al grado de estudios facilitando de alguna manera el aprendizaje de conductas sanitarias. Considerando que la adolescencia es una etapa donde el ser humano adquiere diversos cambios físicos y psicológicos para alcanzar la madurez se debe tomar en cuenta tales características para el entendimiento y trabajo conjunto con dicho grupo de interés frente a su situación de madres responsables de sus hijos de quienes deben velar por su autocuidado.

Con relación al conocimiento de manera global concerniente a higiene alimentaria antes de las intervenciones educativas el $93 \%$ fueron deficientes y $7 \%$ fueron óptimas, después de las intervenciones dio como resultado que el $100 \%$ fueron óptimas. Resultados similares a los de García R, Casado C, Pérez A, Sosa I, quienes obtuvieron que antes de la intervención los estudiantes tenían conocimientos escasos sobre qué son las enfermedades transmitidas por alimentos (47,2\%), así como los agentes causales y los métodos que podrían tomarse para disminuir o erradicar las enfermedades trasmitidas por los alimentos (7). La intervención educativa aplicada demuestra ser eficaz; por tanto, creemos necesario extender las intervenciones educativas a las instituciones escolares de salud en las cuales las enfermedades de transmisión por alimentos constituyen un riesgo para la salud. 
La educación es la base de casi todas las conductas del ser humano y los hábitos saludables también se aprenden. Poco a poco, de forma paulatina, las familias deben inculcar a sus hijos e hijas estos hábitos para que desde pequeños sepan cómo cuidarse y gocen de un estado de bienestar y una buena calidad de vida. La importancia de educar para la buena alimentación, los hábitos de higiene y el cuidado del cuerpo son tres temas en los que se debe hacer especial hincapié. Las técnicas son solo instrumentos o herramientas que están en función de un proceso educativo, en tanto que las metodologías son los sistemas y estilos específicos de adquirir conocimientos en este proceso educativo o de formación y que implican necesariamente una concepción ideológica y /o filosófica que guía el desarrollo de este proceso.

La práctica de higiene alimentaria antes de la intervención educativa fue inadecuada en el $100 \%$ de madres, sin embargo después de la intervención educativa fue adecuado en el $53 \%$ de las madres. Para una adecuada manipulación de los alimentos, se debe comenzar por la selección de dónde comprar los alimentos, para ello se debe tener presente que los establecimientos de venta de alimentos deben estar autorizados y bajo el control e inspección periódica de los Servicios de Salud Pública lo cual es de escaso interés hoy en día dadas las circunstancias económicas. Por ello, la primera garantía de la que debe asegurarse el manipulador es la legalidad del centro donde se adquieren los alimentos y el cumplimiento de las normas de higiene que garanticen la calidad e inocuidad de los diferentes productos alimentarios que se expendan al público usuario. Takanashi $\mathrm{K}$ en su estudio realizado obtuvo como resultados que la higiene de los alimentos, prácticas de las madres, tales como evitar la preparación de alimentos para cocinar en el suelo, tiene un impacto potencial en la prevención de la diarrea entre los niños en Vietnam (9).
Establecer y ejecutar medidas de higiene en nuestro hogar, garantizará que los alimentos no se contaminen y en consecuencia, que no haya daño para la salud de cada miembro de la familia que los consuma. Por otra parte, éstos se conservan mejor y por más tiempo, dentro - por supuesto- de los rangos naturales de cada tipo de alimento. La promoción de estilos saludables de vida y la prevención de enfermedades transmisibles por los alimentos (ETA) es una actividad que se realiza primordialmente en el nivel de atención primaria: centros y puestos de salud (como parte de una descentralización social y de salud); donde la enfermera cumple el rol de educadora, en constante coordinación con el equipo multidisciplinario, para ello realiza actividades educativas en las diferentes organizaciones base de la comunidad.

\section{CONCLUSIONES:}

- El conocimiento sobre higiene alimentaria antes de la intervención educativa fue deficiente sin embargo después de la intervención educativa fue óptima.

- La práctica sobre higiene alimentaria antes de la intervención educativa fue inadecuada sin embargo después de la intervención educativa la práctica mejoró significativamente.

- El efecto de la intervención educativa se demostró a través del estadístico $t$ Student $(4,18)$; por tanto se rechaza la hipótesis nula y se acepta la hipótesis alternativa que afirma que la intervención educativa tuvo efecto positivo en las madres del Centro poblado de Chacarita Sunampe - Chincha provincia de Ica,Perú.

\section{Correspondencia:}

Lic. Viviana Loza Félix

Correo electrónico: vivitaloza@hotmail.com 


\section{REFERENCIAS BIBLIOGRÁFICAS}

1. OMS. Manual sobre las cinco claves para la inocuidad de los alimentos. [Fecha de acceso: 23 de setiembre del 2013]. [Archivo en pdf]. Francia; 2007. Disponible en:

http://www.who.int/foodsafety/publications/ consumer/manual_keys_es.pdf

2. Organización de las Naciones Unidas para la Agricultura y la Alimentación. Políticas de Seguridad e Inocuidad y Calidad Alimentaria en América Latina y el Caribe. [Fecha de acceso: 23 de setiembre del 2013]. [Archivo en pdf]. Chile; 2010. Disponible en: http://www.cvpconosur.org/wpcontent/uploads/2010/08/seguridadalimentaria-2010.pdf

3. MINSA. Morbilidad infantil en consulta externa. [Fecha de acceso: 11 de agosto del 2013]. Lima; 2013. Disponible en http://www.app.minsa.gob.pe/bsc/Detalle_ IndBSC .asp? lcind $=5 \& \mid$ cobj $=1 \&|c p e r=1 \&| c f$ reg=9/4/2013

4. Ministerio de la Mujer y Desarrollo Social. El Programa Nacional Wawa. [Fecha de acceso: 2 de octubre del 2013]. [Archivo en pdf]. Disponible en: http://ebr.minedu.gob.pe/dei/pdfs/semnari o_trujillo_pdf/ponencias/ponencia_propue sta_pedagogica_del_wawawasi.pdf

5. Puente L. Higiene alimentaria. [Tesis de grado]. Cuba; 2010. Disponible en: http://www.portalesmedicos.com/publicaci ones/articles/4181/8/Intervencioneducativa-sobre-habitos-alimentarios-amadres-de-preescolares-con-diarreas-

6. Chaves P. Condiciones higiénico sanitarias de los comedores del mercado municipal de Guaranda. [Tesis de grado]. Ecuador; 2010. Disponible en: http://dspace.espoch.edu.ec/bitstream/123 456789/1684/1/84T00049.pdf
7. García R, Casado C, Pérez A, Sosa I. Intervención educativa sobre enfermedades transmitidas por alimentos en estudiantes de tecnología de la salud en la provincia de Camagüey. [Tesis de grado] Universidad de Ciencias médica de Camagêy Cuba; 2009. Disponible en: http://bvs.sld.cu/revistas/hie/vol50_2_12/hi e10212.htm

8. Matta W; Ortega D. Conocimiento sobre medidas preventivas y prácticas de las madres sobre EDA y su relación con características sociodemográficas [Fecha de acceso: 28 de setiembre del 2013]. [Texto en línea]. http://www.upch.edu.pe/faenf/images/stori es/articulorevista5/madres9

9. Takanashi K. Encuesta de Prácticas de Higiene de los alimentos en el hogar y la diarrea infantil en Hanoi.[tesis maestría], Viet Nam , 2009 http://scielo.sld.cu/scielo.php?script=sci a $\underline{\text { rttext\&pid }=S 1561-30032012000200010}$

Recibido: 25/07/2014

Aprobado para publicación: 27/11/2014 